\title{
Measurement of Thermal and Epithermal Neutron Flux Distribution in the Torus Hall of LHD using Activation Method in the First Deuterium Experiment Campaign*)
}

\author{
Tomoyo TANAKA, Makoto KOBAYASHI ${ }^{1,2)}$, Sachiko YOSHIHASHI, Akira URITANI, \\ Kenichi WATANABE, Atsushi YAMAZAKI, Takeo NISHITANI ${ }^{1)}$, Kunihiro OGAWA ${ }^{1,2)}$ \\ and Mitsutaka ISOBE ${ }^{1,2)}$ \\ Nagoya University, Nagoya 464-8603, Japan \\ ${ }^{1)}$ National Institute for Fusion Science, National Institutes of Natural Sciences, Toki 509-5292, Japan \\ ${ }^{2)}$ SOKENDAI (The Graduate University for Advanced Studies), Toki 509-5292, Japan
}

(Received 10 January 2019 / Accepted 19 September 2019)

\begin{abstract}
In the Large Helical Device (LHD) of the National Institute for Fusion Science, a deuterium plasma experiment was performed in March 2017. Neutrons with an energy of $2.45 \mathrm{MeV}$ are generated by d $(\mathrm{d}, \mathrm{n}){ }^{3} \mathrm{He}$ reactions. The evaluation of this neutron flux in the torus hall, as well as within the LHD vessel, is very important for the decommissioning of the LHD in the future and the radiation shielding for workers and components around the LHD. In this study, we evaluate the spatial distribution of thermal and epithermal neutron flux on the floor level of the LHD torus hall by measuring the neutron activation of gold foils. The flux information obtained in the experiment can be used for the evaluation of radio-activation and the shielding design.
\end{abstract}

(c) 2019 The Japan Society of Plasma Science and Nuclear Fusion Research

Keywords: LHD, neutronics, activation foil, IP, HPGe

DOI: $10.1585 /$ pfr.14.3405162

\section{Introduction}

At the National Institute for Fusion Science, deuterium plasma experiments at the Large Helical Device (LHD) are performed in order to obtain fundamental data for the design of nuclear fusion power plants. In commercial fusion reactor, neutrons generated by the nuclear fusion reaction will be utilized for thermal energy in fusion power generation. On the other hand, neutrons activate devices within fusion reactors that are composed of several materials. Therefore, it is important for the development of nuclear fusion reactors to understand the behavior of neutrons for radiation protection and for future decommissioning measures.

The first deuterium plasma experiment was performed from March to July of 2017 at the LHD [1]. Neutrons with an energy of $2.45 \mathrm{MeV}$ are generated by the $\mathrm{d}(\mathrm{d}$, n) ${ }^{3} \mathrm{He}$ reaction. These fast neutrons are scattered by the surrounding structures of the LHD, are decelerated, and thus become thermal and epithermal neutrons. As a result, a large number of radioactive isotopes are produced in the torus hall. Many radioactive isotopes have specific decay processes and decay rates and long half-life; therefore, it is necessary to predict the radioactive isotopes that are produced in the torus hall of the LHD for its decom-

author'se-mail: s-yoshihashi@energy.nagoya-u.ac.jp

*) This article is based on the presentation at the 27th International Toki Conference (ITC27) \& the 13th Asia Pacific Plasma Theory Conference (APPTC2018). missioning. A three-dimensional Monte Carlo simulation is typically used to predict the neutron behavior, and the General Monte Carlo N-Particle Transport Code version 6 (MCNP6) has been applied for neutronics of LHD [2,3]. In order to confirm the validity of the simulation, experimental evaluation is necessary. The first measurement of the thermal neutron flux distribution on the floor level of the LHD torus hall was carried out using indium foils [4]. However, the neutron population in the LHD torus hall are included not only thermal neutrons but also epithermal and fast neutrons. The neutron flux of each energy range needs to be evaluated because the radioactive isotopes generate depending on the specific neutron energy.

In this study, we measured the spatial distribution of neutron flux on the floor level of the LHD torus hall by the neutron activation of gold foils. In order to evaluate the thermal and epithermal neutron distributions separately, gold $\mathrm{Au}$ ) foils were covered with cadmium, which acted as a thermal neutron absorber. We put gold foils with and without cadmium covers on the floor level of the torus hall. After the gold foil atoms were activated by the ${ }^{197} \mathrm{Au}$ (n, $\gamma){ }^{198} \mathrm{Au}$ reaction, the gamma rays emitted by gold foils were measured by a high purity germanium (HPGe) detector and an imaging plate (IP). The flux obtained in the experiment will be an important benchmark in the safety evaluation of deuterium experiments in the LHD. 


\section{Experimental Setup}

\subsection{Activation foil}

The stable isotope ${ }^{197} \mathrm{Au}$, whose natural abundance is $100 \%$, was selected for the activation foil in this work. ${ }^{197} \mathrm{Au}$ produces the radioactive isotope ${ }^{198} \mathrm{Au}$ (half-life of about 2.7 days [5]) by a neutron capture reaction, (n, $\gamma$ ) reaction, whose cross section is large for the thermal neutron.

The radioactivity of the activation foil does not directly indicate the neutron fluence at the position of the foil because the radioactive isotopes in the activation foil decay during further neutron irradiation. The quantities of the radioactive isotopes produced during neutron irradiation can be calculated simply by the following equation:

$$
\frac{d N^{(A)}}{d t}=N^{(S)} \sigma \phi-\frac{\ln 2}{T_{1 / 2}} N^{(A)} .
$$

Here, $N^{(A)}$ and $N^{(S)}$ are the quantities of radioactive and stable isotopes, respectively, $\phi$ presents the neutron flux $\left[\mathrm{cm}^{-2} \mathrm{~s}^{-1}\right]$, and $T_{1 / 2}$ is the half-life [s]. The reaction cross section, $\sigma\left[\mathrm{cm}^{2}\right]$ is dependent on the neutron energy. Here, $N^{(A)}$ is zero before the neutron irradiation. The halflife of ${ }^{198} \mathrm{Au}$ is discribed above. Note that $\phi$ is different in each deuterium plasma operation and that $N^{(A)}$ is determined by the integration of the different neutron fields. On the other hand, the position of the site of neutron generation and the energy $(2.45 \mathrm{MeV})$ are almost the same in all deuterium plasma operations.

The dimensions of the gold foils used for the measurement were $0.5 \times 0.5 \mathrm{~mm}^{2}$ with a thickness of $0.1 \mathrm{~mm}$. In addition, in order to measure the epithermal neutron flux, cadmium covers with a thickness of $1 \mathrm{~mm}$ were prepared and acted as thermal neutron absorbers.

\subsection{Components in the LHD torus hall}

The equipment layout in the torus hall of the LHD is shown in Fig. 1. The torus hall dimensions are W75, $\mathrm{L} 45, \mathrm{H}_{40} \mathrm{~m}^{3}$, and the walls of the torus hall are composed of plain concrete with a thickness of $2 \mathrm{~m}$. The LHD is equipped with many systems for plasma heating, plasma diagnosis, and other purposes. In addition to the spatial neutron detection, the neutron yield also was measured by neutron flux monitors a ${ }^{235} \mathrm{U}$ fission chamber, ${ }^{10} \mathrm{~B}$ counters and ${ }^{3} \mathrm{He}$ counters, positioned above the vicinity of LHD central axis of the large external port [6]. Unfortunately, the ${ }^{10} \mathrm{~B}$ counters and ${ }^{3} \mathrm{He}$ counters were saturated with high neutron flux, so the neutron generation rates measured by these counters are not used in this paper.

Borated polyethylene blocks are used in the LHD as neutron shielding. Polyethylene can effectively decelerate fast neutrons to thermal neutrons due to their light constituent atoms. The boron in the polyethylene blocks can also absorb thermal neutrons, due to the large cross section of the ${ }^{10} \mathrm{~B}(\mathrm{n}, \alpha){ }^{7} \mathrm{Li}$ reaction. These borated polyethylene blocks contain $10 \mathrm{wt} \%$ boron. A number of electrical components within the torus hall were covered with borated polyethylene blocks in order to suppress any influence or

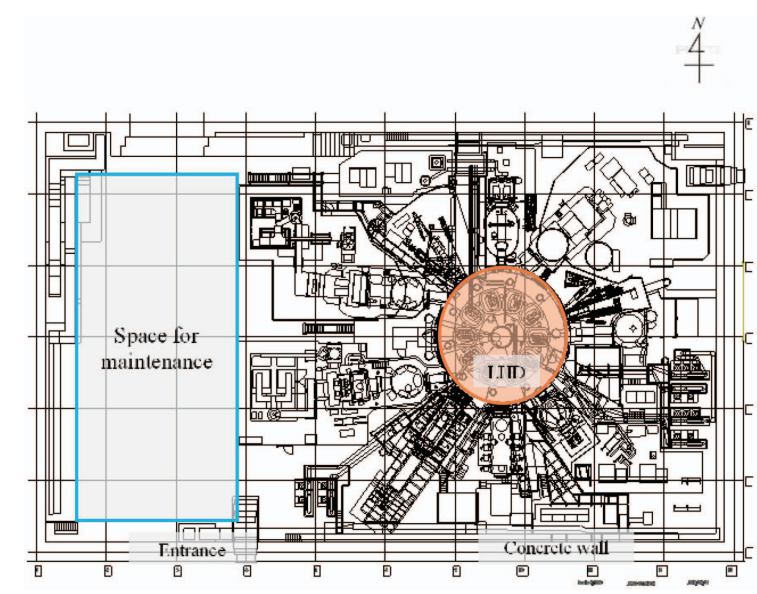

Fig. 1 Component layout of the LHD torus hall.

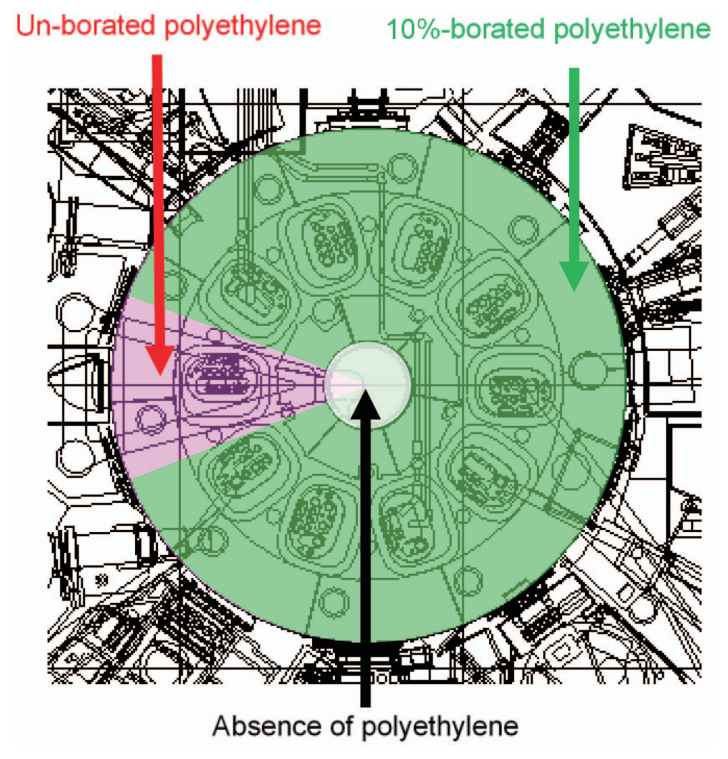

Fig. 2 Layout of polyethylene blocks under the LHD floor.

activation from the neutrons. As shown in Fig. 2, the borated polyethylene blocks were placed in a disk-like shape with an inner diameter of $2.3 \mathrm{~m}$, an outer radius of $6.9 \mathrm{~m}$, and a thickness of $5 \mathrm{~cm}$ on the floor underneath the LHD. However, under the west side of the LHD, an unborated polyethylene block was placed instead.

\subsection{Neutron irradiation and gamma ray analysis}

As shown in Fig. 3, the prepared gold foils were placed on the floor of the torus hall and left for about two weeks during the deuterium experiment. Around 500 shots were carried out, and the total neutron generation measured by the ${ }^{235} \mathrm{U}$ fission chamber was around $1.0 \times 10^{16}$ neutrons.

The gamma-rays emitted by ${ }^{198} \mathrm{Au}$ were measured using two analytical methods: HPGe detector and IP. The HPGe detector is capable of identifying and evaluating the exact amounts of radioactive isotopes by gamma-ray energy spectroscopy and is widely used in the field of radi- 


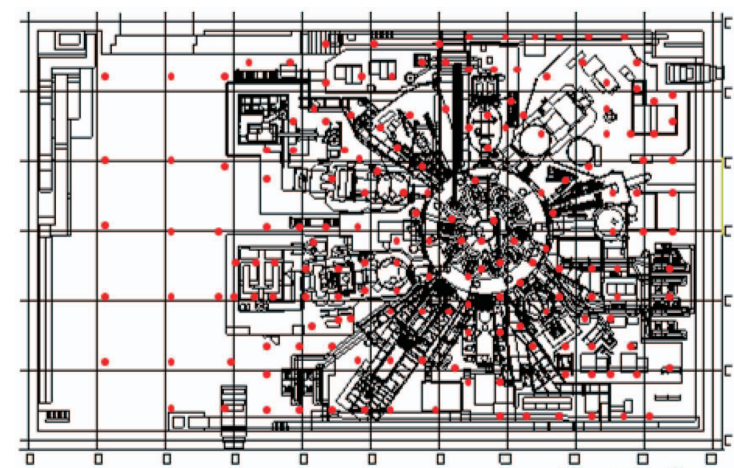

Fig. 3 Foil position on the floor of the LHD.

ation management. However, as this method usually can measure only one sample at a time, a long measuring time would be required if all the foils were to be analyzed this way. Therefore, we used an IP to measure many samples at a time. IP is a sheet-type analytical method that accumulates energy transferred from radiation. IP can release accumulated energy as photostimulated luminescence (PSL) by laser injection. As IP usually has the ability to measure a large area, it is applied to the two-dimensional visualization of radiation sources. The samples were stuck onto Mylar film, with a thickness of $100 \mu \mathrm{m}$, and then placed on the IP. After that, IP measurement was performed to all samples. A small number of samples were removed from the Mylar film and measured by the HPGe detector for gamma-ray spectroscopy. Details of these analyses are described in Ref. [7].

\section{Results and Discussion}

\subsection{Neutron flux measurement}

An example of the gamma-ray spectrum emitted by the gold foil measured by the HPGe detector is shown in Fig. 4. A dominant $412 \mathrm{keV}$ peak from ${ }^{198} \mathrm{Au}$ can be seen.

In order to confirm the relationship between the neutron flux calculated by the $412 \mathrm{keV}$ gamma-ray count by the HPGe detector and the PSL measured by the IP, the two measurement results were compared, for foils placed in three places in Fig. 5. It was confirmed the linearity between the neutron flux calculated from the HPGe detector and radiation intensity measured with IP. Although the IP detected both gamma rays and beta rays, the released PSL was predominantly $412 \mathrm{keV}$ gamma rays.

\subsection{Neutron flux distribution}

Figure 6 shows (a) the thermal neutron flux distribution and (b) the epithermal neutron flux distribution on the floor level of the LHD torus hall. Here, $\phi$ indicates the neutron flux $\left[\mathrm{cm}^{-2}\right.$ source $\left.{ }^{-1}\right]$ from single neutron generated in the total plasma volume of the LHD vacuum vessel. The thermal neutron flux distribution was obtained by subtracting the radioactivity of the cadmium-covered gold foils from that of those not covered with cadmium. The ep-

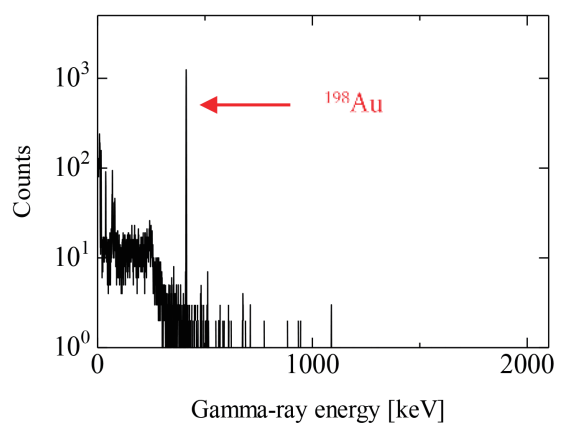

Fig. 4 An example of the gamma-ray spectrum of gold foil measured by the HPGe detector.
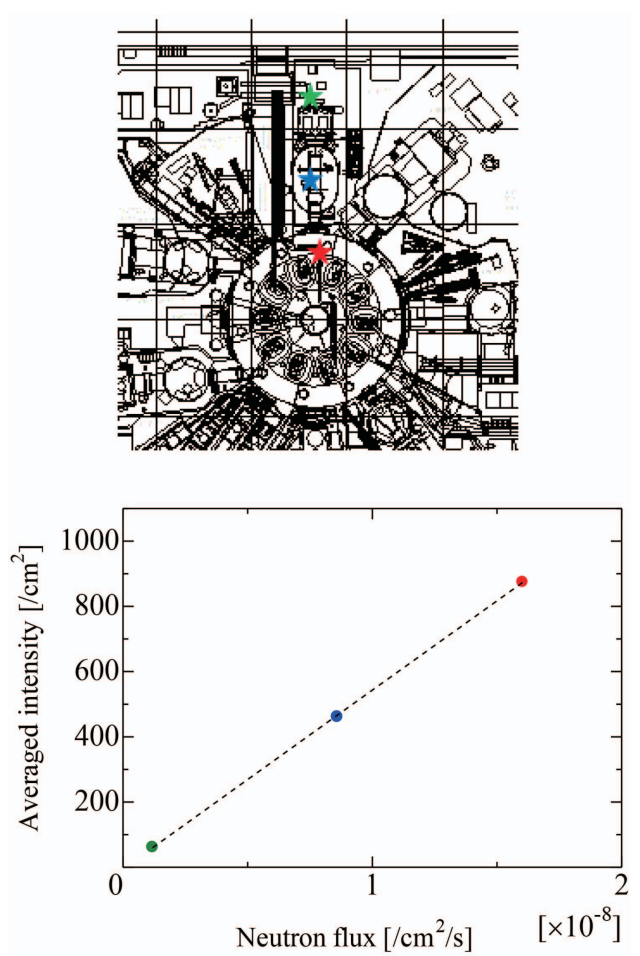

Fig. 5 The relationship the neutron flux calculated using the HPGe detector and radiation intensity measured by the IP at three positions near the LHD.

ithermal neutron flux distribution was found by the result of gold foils covered with cadmium. These figures were created using the Generic Mapping Tool version 5.4.3 [8]. Although the distribution is blurred because of the distance between samples, an overall trend in the neutron distribution can be seen. The thermal neutron flux was relatively high in the vicinity of the LHD and is a similar result to that obtained when using the ${ }^{155} \mathrm{In}$ (n, g) ${ }^{116 \mathrm{~m}}$ In reaction [4]. Figure 6 (a) shows that a higher thermal neutron flux was observed in the west side of the LHD, which shows the distribution of thermal neutrons well. The maximum thermal neutron flux in Fig. 6 (a) is $4.04 \times 10^{-8} \mathrm{~cm}^{-2}$ source $^{-1}$.

On the other hand, it was observed that the epithermal neutron flux was relatively high in the center of the LHD, $2.76 \times 10^{-8} \mathrm{~cm}^{-2}$ source ${ }^{-1}$ and that is uniformly decreased outwards to $2.54 \times 10^{-10} \mathrm{~cm}^{-2}$ source $^{-1}$. 


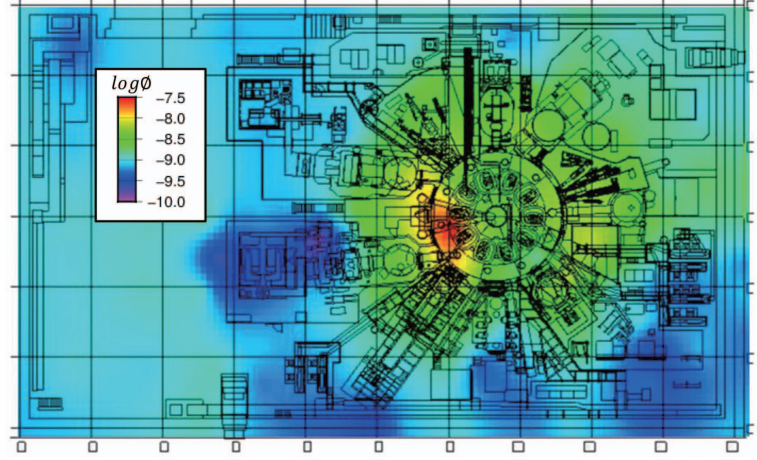

(a) thermal neutron flux distribution

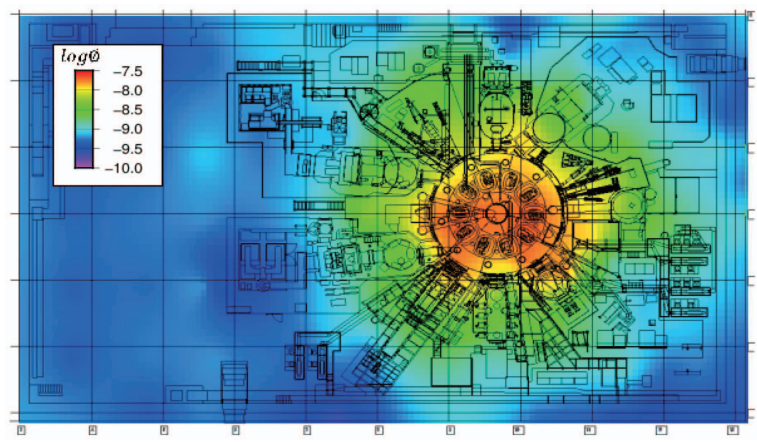

(b) epithermal neutron flux distribution

Fig. 66 The neutron flux distributions $\Phi$ on the floor level of the LHD torus hall (a) thermal neutrons measured by $\mathrm{Au}$ foils, (b) epithermal neutrons measured by Au foils with Cd filter.

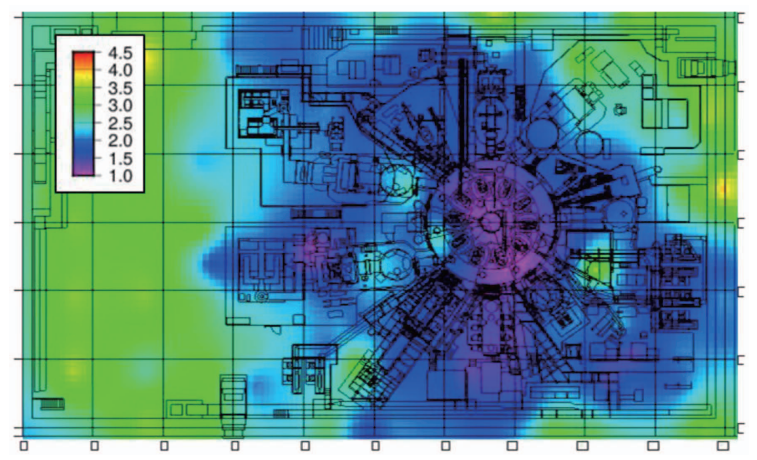

Fig. 7 The distribution of the cadmium ratio of the thermal neutron flux on the floor level of the LHD torus hall.

Figure 7 shows the distribution of the cadmium ratio on the floor level of the LHD torus hall. The cadmium ratio is defined by the following equation.

$$
\text { Cadmium ratio }=\frac{\Phi_{A u}}{\Phi_{A u+C d}} .
$$

Here, $\phi_{A u}$ and $\phi_{A u+C d}$ represent the neutron flux given by the gold foil and the cadmium-covered gold foil, respectively. The fast neutrons generated in the LHD are moderated to thermal or epithermal neutrons by the components within the LHD torus hall. The cadmium ratio shows to what extent the thermal neutrons dominate at each mea- surement position. In other words, although fast neutrons cannot be measured in this experiment, it is possible to know how much they have been moderated by the LHD components. In Fig. 7, the cadmium ratio was low near the LHD and increases at greater distances from the center of the LHD. This implies that the fast neutrons were effectively decelerated by the borated polyethylene block under the floor. In addition, the cadmium ratio was relatively large at the place where the unborated polyethylene block was located, under the west side of the LHD. This confirms that, since boron was not present in the block, the fast neutrons were not effectively converted into thermal neutrons. In addition, it shows the influence of the polyethylene block installed near the area of large cadmium ratio. These measurement results show the effect of the moderation by the components around the LHD well and can be expected to be useful for the future shielding design.

\section{Conclusion}

The thermal and epithermal neutron fluxes on the floor level of the LHD torus hall were experimentally evaluated in order to estimate the neutron field generated by deuterium plasma operation in the LHD.

Gold activation foils were used, and the radioactivity of these foils was evaluated with IP and HPGe detector. By using gold foils covered with or without cadmium, we could obtain spatial distributions of the thermal and epithermal neutron flux. A relatively high thermal neutron flux was observed on the west side of the LHD, whereas a significant epithermal neutron flux was not observed around the outside of the LHD. The difference in those flux distributions is likely caused by the combination of polyethylene blocks and boron that acted as a fast neutron decelerator and thermal neutron absorber. The neutron flux distribution evaluated in this work will be used for the evaluation of radioactive waste in the torus hall and neutron shielding designs.

\section{Acknowledgment}

This work was supported by the LHD project budget (ULGG801 and ULAA027). T. Tanaka is pleased to acknowledge the LHD Experiment Group for assistance in activation foil measurement.

[1] Y. Takeiri et al., IEEE Trans. Plasma Sci. 46, 2348 (2018).

[2] X-5 Monte Carlo Team, MCNP User's Guide - Code Version 6.1.1beta, LA-CP-14-00745 (Los Alamos National Laboratory, Los Alamos, 2014).

[3] T. Nishitani et al., Prog. Nucl. Sci. Technol. 6, 48 (2019).

[4] M. Kobayashi et al., Fusion Eng. Des. 137, 191 (2018).

[5] R.B. Firestone, Table of Isotopes, 8th edition (John Wiley \& Sons, Inc., 1996).

[6] M. Isobe et al., Rev. Sci. Instrum. 85, $11 \mathrm{E} 114$ (2014).

[7] M. Kobayashi et al., Prog. Nucl. Sci. Technol. 6, 58 (2019).

[8] P. Wessel and W.H.F. Smith, EOS Trans. Amer. Geophys. U. 94, 409 (2013). 